\title{
The life course, childhood housing conditions and adult health
}

Socioeconomic conditions are known to be major determinants of health at all stages of the life course from pregnancy, ${ }^{1}$ childhood, ${ }^{2}$ and adulthood. ${ }^{3}$ Life course epidemiology has added a further dimension to our understanding of the social determinants of health showing an association between early socioeconomic conditions and adult morbidity and mortality, ${ }^{4}$ and adult health related behaviours. ${ }^{5}$ Differential exposures over the life course are also likely to partly account for social gradients in health with the cumulative and additive effects of risk and protective factors exerting their effects over time. ${ }^{6}$

The causal debate related to the social determinants of health has been profoundly altered by recognition of the life course effects of early socioeconomic conditions. Single exposures, such as smoking, measured at a single time point can no longer be held to "account for" social differences in health. Multiple exposures operating over time seem to be implicated in the pathways from socioeconomic conditions to health outcomes.

Housing conditions, particularly overcrowding, poor repair, and poor sanitation, are important components of socioeconomic conditions and have been linked historically with adverse health outcomes. Housing tenure (owner occupation versus rented) remains a powerful proxy for household income in the UK. The proportion of rented and overcrowded households in an area are components of a number of census-based area deprivation indices used extensively in the UK. Intuitively, housing is associated with health and wellbeing and is consistent with the observation that, at least in the UK, those with higher incomes gravitate to less crowded accommodation in a better state of repair. However, clear evidence of an effect of housing conditions on health independent of other socioeconomic variables remains elusive.

Dedman et al in this issue show that childhood housing conditions have only a weak association with mortality in adulthood after adjustment for childhood and adult socioeconomic factors. In view of the lack of clear evidence of an independent effect of current housing conditions on current morbidity or mortality, it is perhaps not surprising that childhood housing conditions exert only a weak influence on adult mortality. As the authors point out, the use of an area-based measure of adult socioeconomic status may inadequately control for this potential confounder. However, elimination of any resulting residual confounding would be likely to further weaken the already weak relation between housing conditions and mortality. By contrast, the selection of the initial sample to include poorer sections of the British population is likely to have introduced a bias towards poorer housing conditions with the result that the "worst" conditions could not be compared with the "best".

This paper is a useful further contribution to the life course literature particularly as it seeks to investigate whether a specific aspect of poor childhood socioeconomic conditions, in this case housing conditions, is related to subsequent adult mortality. Identification of an independent association between a specific early exposure and a specific cause of adult mortality is likely to lead to a more precise understanding of the mechanisms by which early childhood conditions influence adult health and, in addition, may have policy implications. The observed association of lack of a private tapped water supply with coronary heart disease mortality is a case in point although the association is relatively weak and further research will be required to elucidate the link.

The attempt to identify associations between specific early exposures and specific causes of adult mortality raises an interesting methodological challenge. If the pathways from socioeconomic conditions to adult health outcomes entail multiple exposures over time as suggested above, can the complexities of these relations be adequately examined using multiple regression modelling? Multiple regression models do not make explicit the temporal relations in life course pathways. They may also conceal indirect effects of distal exposures working through proximal exposures on the outcome. ${ }^{8}$ Path analysis has been proposed as an alternative method for exploring pathways characterised by multiple exposures over time. ${ }^{9}$ Although not without its own methodological limitations, ${ }^{9}$ path analysis seems to offer an instrument capable of further elucidating life course pathways.

Greater understanding of the social determinants of health and the role of early life exposures in later morbidity and mortality is likely to come from further exploration of social gradients and the way in which the effects of multiple exposures exert their influence over time. The paper by Dedman $e t a l^{7}$ adds to the growing life course epidemiology literature. Progress in examining the effects of specific early exposures may require the more widespread use and development of methodologies, such as path analysis, capable of accounting for indirect effects of distal exposures.

Department of Applied Social Studies/School of Post NICK SPENCER

School of Postaduate Medical

Education, University of Warwick, Coventry CV4 7AL, UK

(n.j.spencer@warwick.ac.uk)

1 Stern A, Campbell EA, Day A, et al. Social adversity, low birth weight, and preterm delivery. BMF 1987;295:291-3.

2 Spencer NJ. Poverty and child health. Oxford: Radcliffe Medical Press, 1996. 3 Marmot M, Wilkinson RG, eds. Social determinants of health. Oxford: Oxford University Press, 1999.

4 Kuh D, Power C, Blane D, et al. Social pathways between childhood and adult health. In: Kuh D, Ben-Shlomo Y, eds. A life course approach to chronic disease epidemiology. Oxford: Oxford Medical Publications, 1997.

5 Lynch JW, Kaplan GA, Salonen JT. Why do poor people behave poorly? Variation in adult health behaviours and psychosocial characteristics by stages of the socioeconomic lifecourse. Soc Sci Med 1997;44:809-19.

6 Blane D, Bartley M, Davey Smith G. Disease aetiology and materialist explanations of socioeconomic mortality differentials. Eur $\mathcal{F}$ Public Health 1997;7:385-91.

7 Dedman DJ, Gunnell D, Davey Smith G, et al. Childhood housing conditions and later mortality in the Boyd Orr cohort. F Epidemiol Community Health 2001;55:10-15.

8 Sheehan TJ. Stress and low birth weight: a structural modeling approach using real life stressors. Soc Sci Med 1998;47:1503-12.

9 Susser M, Levin B. Ordeals for the fetal programming hypothesis. BMf 1999;318:885-6. 